\title{
CERAMIC MATERIALS CHARACTERIZATION USING MINIATURE MECHANICAL TESTS: COMPARISON BETWEEN B3B AND SPT TESTS
}

\author{
C. Quintana ${ }^{1}$, C. Rodríguez ${ }^{1}$, F.J. Belzunce ${ }^{1}$, A.C. Caballero ${ }^{2}$, C. Baudín ${ }^{2}$ \\ ${ }^{1}$ SIMUMECAMAT Research group, Universidad de Oviedo. Edificio Departamental \\ Oeste, Bloque 7. Campus Universitario. 33203 Gijón, Spain \\ ${ }^{2}$ Instituto de Cerámica y Vidrio, CSIC. Kelsen 5, 28049 Madrid, Spain
}

\begin{abstract}
Strength of ceramic components is determined by processing, therefore, test methods that allow analysing their actual mechanical behaviour are needed. The miniature mechanical tests ball three balls (B3B) and small punch (SPT) are compared using commercial lead zirconate titanate (PZT) piezo-ceramic discs. The Weibull distributions of strength were obtained and the fracture surfaces were observed by scanning electron microscopy. The different approaches for the calculation of strength values are discussed.

The adequacy of the small punch test for the determination of strength is demonstrated. Results for SPT and B3B tests are comparable considering the effective volumes calculated using the Weibull relationships.

The Timoshenko equation for strength is proposed for the calculus of strength due to its simplicity and the fact that the calculated values for B3B and SPT geometries are not dependent on the radius of the uniform loading central area.
\end{abstract}

KEYWORDS: Miniature strength tests, SPT, B3B, PZT, Weibull

\section{Introduction}

Nowadays, materials based on $\mathrm{Pb}\left(\mathrm{Zr}_{\mathrm{x}} \mathrm{Ti}_{1-\mathrm{x}}\right) \mathrm{O}_{3}, \mathrm{PZT}$, are the most important commercial piezo-ceramics because their piezoeletric performance is better than that of any other material. PZT's are the constituents of a large number of components with 
a wide variety of shapes and sizes: monolithic devices, multilayer actuators, integrated microelectromechanical systems (MEMS) ... etc. The piezoeletric properties strongly depend, not only on the microstructure of the materials, but also on the specific device geometry required to fit the application needs $[1,2]$. For instance, it is well known that, among other parameters, final geometry of piezoceramic devices is fundamental in the material ageing process. Microstructure and geometry are determined by the specific processing procedure.

The failure behavior of piezoelectric ceramics under combined mechanical and electrical loads is complex. Electrical loading might lead to high energy dissipation during crack propagation due to an electrical "plastic deformation," such as electric discharge and domain switching. However, the fracture mode of piezoelectric ceramics under pure mechanical loading, is brittle, and the most used property to characterize their fracture behavior is strength $[3,4]$.

Piezo-ceramic materials are prone to aging due to the high mechanical stresses originated by the electric domain switching during the poling process and under working conditions. Computer models and simulations might help to understand and eventually predict ageing and material failure. In this regard, for accurate simulations it is necessary to count with precise data of the material response. In this sense, the characterization of real parts is sought to describe the polarised material behaviour. According to this, it would be possible to achieve a great advance in this field, if the mechanical response of PZT's could be characterized by experimental procedures that allow employing real industrial component as test specimens.

This is the case of the "Ball-on-three-balls" (B3B) test, especially developed for the determination of the fracture stress of miniature ceramic components [5]. This test system, barely sensitive to geometric imprecision [6], was designed in order to test "as sintered" discs without the necessity of machining.

Figure 1 shows a schema of the B3B jig [6]. It consists of a four ball system, where three balls act as supports for the disc shaped specimen and a fourth ball applies the load on the opposite face of the specimen. The three supporting balls are in contact with each other forming an equally sided triangle of support points. During the test, the 
specimen is free to move and no friction occurs. A more detailed description of this test can be found elsewhere [5-9].

It has been demonstrated that the $\mathrm{B} 3 \mathrm{~B}$ test gives accurate strength values for different ceramic materials [5-9], being its main weakness the difficulty of handling the four small balls.

For homogeneous and isotropic materials, fracture will start from the zone of maximum tensile stress. Assuming lineal elastic behaviour, the stress distribution analysis of the B3B test [5-10]shows that the maximum tensile stress is located in the central part of the face of the disc subjected to tension (upper face of the disc in Fig. 1a).

Even though the B3B test is quite commonly used in the ceramic community, the calculation of the maximum stress, $\sigma_{c}$, is still under controversy. It is accepted the general relationship between $\sigma_{c}$ and the fracture load, $P_{c}$, and the specimen thickness, $t$, given by Eq. (1):

$$
\sigma_{c}=f \cdot \frac{P_{c}}{t^{2}}
$$

However, there are different proposals to obtain the value of the proportionality factor, $f$, which depends on different geometrical parameters -specimen radius, $R$; radius of the loading ball, $R_{b}$; support radius, $R_{a}$; specimen thickness, $t$; and radius of the central loaded area, which is assumed subjected to a constant stress value, $b-$, as well as on material factors -Poisson's coefficient and Young's modulus of both, balls $\left(v_{b}, E_{b}\right)$ and tested material $(v, E)$-.

By applying the linear-elastic theory to thin plates subjected to a central constant stress acting on a circular area with radius $b$, different authors reach different analytical expressions for the proportionality factor, f: Eq. (2), by Xiong et al. [11]; Eq. (3), by Soltysiak et al. [12]; Eq. (4), by Timoshenko et al. [13]; and Eq. (5), by Shetty et al. [14].

$$
\begin{aligned}
& f=\frac{3}{2 \pi}\left[1-\frac{1-v^{2}}{4} \frac{b^{2}}{R_{a}^{2}}+(1+v) \ln \frac{R_{a}}{b}\right] \\
& f=\frac{3}{2 \pi}\left[1+(1+v) \ln \frac{R_{a}}{b}\right]
\end{aligned}
$$




$$
\begin{aligned}
& f=(1+v)\left[0.485 \ln \left(\frac{R_{a}}{t}\right)+0.52\right]+0.48 \\
& f=\frac{3(1+v)}{4 \pi}\left[1+2 \ln \frac{R_{a}}{b}+\frac{(1-v)}{(1+v)}\left(1-\frac{b^{2}}{2 R_{a}^{2}}\right) \frac{R_{a}^{2}}{R^{2}}\right]
\end{aligned}
$$

Except of Eq. (4), all expressions require knowing the value of the uniformly loaded central area, $b$, for which different proposals are found in the scientific literature, as shown in Table 1.

For Shetty et al. [14], $b$ only depends on the thickness of the specimen, while Westergard [15] includes the contact radius between the loading ball and the specimen, $\zeta$, which can be approximated by [16]:

$$
\zeta=\left(\frac{3 P R_{b}}{4 E^{\prime}}\right)^{1 / 3}
$$

Being $P$ de applied load and $E^{\prime}$, the reduced Young's modulus:

$$
\frac{1}{E^{\prime}}=\frac{\left(1-v_{b}^{2}\right)}{E_{b}}+\frac{\left(1-v^{2}\right)}{E}
$$

The numeric approach by Börger et al. [5] to calculate the parameter $f$, based in finite element modelling (FEM), gives the relationship of equation (8) in which the values of the seven coefficients $\left(c_{0}, c_{1}, c_{2}, c_{3}, c_{4}, c_{5}, c_{6}\right)$ depend on the Poisson's coefficient of the analysed material.

$$
f=c_{0}+\frac{\left(c_{1}+c_{2} \frac{t}{R}+c_{3}\left(\frac{t}{R}\right)^{2}+c_{4}\left(\frac{t}{R}\right)^{3}\right.}{1+c_{5} \frac{t}{R}}\left(1+c_{6} \frac{R_{a}}{R}\right)
$$

The "Small punch test" (SPT) is a miniature biaxial test as the B3B that was initially designed for testing irradiated steel samples [17]. Figure 2.a) shows the SPT configuration used for steel testing [18]. A disc shaped specimen (usually $10 \mathrm{~mm}$ diameter and $0.5 \mathrm{~mm}$ thickness) is clamped between two rings and deformed by the action of a hemispherical punch that forces the specimen to deform through the internal 
hole of the supporting ring. This test configuration is widely accepted for the mechanical characterization of steel and other metallic alloys [18-21] and its applicability has been extended to other types of materials like polymers $[22,23]$.

Unlike the B3B test, the SPT is barely used by the ceramic community. Little information is available in the literature $[8,11,12]$ and the experimental details -sizes of the specimens and supports and specimen clamping- vary widely. In addition, different ways of fracture stress calculation are reported. Most researchers agree that for the characterization of brittle materials it is advantageous not to clamp the specimen to prevent initial stresses due to small geometrical misalignments between the specimen and the bearing $[8,12]$. Figure $2 . b)$ shows schematically this type of configuration in which the specimen is simply supported on the supporting ring.

As equations (2-5) refer to thin plates subjected to uniform stresses acting on circular areas, they are also adequate to determine strength in the case of testing small discs using the SPT. In addition, the SPT allows estimating other material properties such as yield stress and Young's modulus $[18,19,21,24]$.

The aim of this study is to compare the B3B and the SPT tests for the characterisation of strength of PZT materials in order to evaluate the adequacy of the later for testing ceramic components. Special care has been taken to analyse the different approaches for the calculation of strength values (Eqs. 2-5 and 8) in order to identify the most adequate equations for the calculation of B3B and SPT strength values.

\section{Experimental}

\subsection{Material and mechanical tests}

Test specimens were commercial discs of soft PZT (NCE 51, Noliac, Czech Republic) of $12.66 \mathrm{~mm}$ diameter and $1 \mathrm{~mm}$ thickness. This geometry is the typical of small monolithic sensors/actuators for different circuit applications. According to the literature, properties of this kind of piezo-ceramics are expected to be in the following ranges: Poisson's coefficient, $v \approx 0.3$ [25], Young's modulus , $E=70-100 \mathrm{GPa}[26,27]$, and four-point bending Weibull characteristic strength values, $\sigma_{0}=80-115 \mathrm{MPa}$ [28-30]. 
The B3B tests were performed using a special device developed by the University of Leoben (Austria) $[5,6]$ installed in a static testing machine (INSTRON 4443, Great Britain) of $1 \mathrm{KN}$ load capacity, using a load cell of 500N maximum capacity and a recording sampling frequency of $10 \mathrm{~Hz}$. The balls, made of martensitic stainless steel $(v=0.33$ and $E=200 \mathrm{GPa}$ ), have a radius of $R_{b}=4.25 \mathrm{~mm}$. As the support points set up an equally sided triangle, the loading radius, $R_{a}$, is $4.91 \mathrm{~mm}\left(\mathrm{R}_{\mathrm{a}}=\frac{2 \sqrt{3}}{3} \mathrm{R}_{\mathrm{b}}\right)$.

The SPT tests were performed using a device specially designed by the SIMUMECAMAT research group (University of Oviedo, Spain) [18] installed in a static testing machine (MTS SMT3-166) with $10 \mathrm{KN}$ of load capacity and using a load cell of $5 \mathrm{KN}$ and recording sampling frequency of $20 \mathrm{~Hz}$. The supporting ring, made of high strength steel, has an internal diameter of $4 \mathrm{~mm}$. The punch, with a diameter of $2.5 \mathrm{~mm}$, is made of martensitic stainless steel ( $v=0.33$ and $E=200 \mathrm{GPa}$ ). SP tests were done using the unclamped configuration (Fig. 2.b), as suggested by most researchers for brittle materials.

24 specimens were tested in each test configuration, with the positive polarized side in tension, and using a constant displacement rate of $0.1 \mathrm{~mm} / \mathrm{min}$. The failure loads, $P_{c}$, were extracted from the load-displacement curves recorded during the tests and the strength values, $\sigma_{c}$, were determined from the failure loads (Eq. 1) using the different expressions for the factor, $f$, for comparative purposes (Eqs. 2-5, 8). "As fractured" surfaces were observed by scanning electron microscopy (SEM, Hitachi TM-1000, Japan).

The Weibull distributions of the simplified strength values, $\sigma=P_{c} / t^{2}$, for both series of specimens were calculated according to the European standard EN 843-5 (2006) [31]. This standard considers a two-parameters Weibull distribution [32] in which the probability of failure, $P_{f}$, for a given stress, $\sigma$, is given by equation (9):

$$
P_{f}(\sigma)=1-\exp \left(-\left(\frac{\sigma}{\sigma_{0}}\right)^{m}\right)
$$

To determine the characteristic strength, $\sigma_{0}$ (stress for a failure probability of $63.2 \%)$, and the Weibull modulus, $m$, the experimental values of strength are arranged 
in ascending order and a failure probability given by Eq. (10) is associated to each experimental $\sigma_{i}$ value, where $N$ is the total number of specimens tested and, $i$, is the order number of the strength.

$$
P_{f}\left(\sigma_{i}\right)=\frac{i-0.5}{N}
$$

Then, $\sigma_{0}=\left(P_{c} / t^{2}\right)_{0}$ and $m$ are determined analytically using the maximum likelihood principle. The upper and lower limits for the $90 \%$ confidence intervals of $\sigma_{0}=\left(P_{c} / t^{2}\right)_{0}$ and $m$ were calculated using the UNE-EN 843-5:2006 proposed method [31].

\subsection{Comparison between strength results for SPT and B3B}

Results of different strength tests can only be directly compared when stress states are similar and the volumes of specimen under the maximum tensile stresses (i.e: effective volumes) are coincident [33]. Using the parameters of the $\sigma_{c}$ distributions, the effective volumes for both considered tests were calculated using Eq. (11):

$$
P_{f}(\sigma)=1-\exp \left(-\frac{V_{e f f}}{V_{0}}\left(\frac{\sigma}{\sigma_{0}}\right)^{m}\right)
$$

Where $V_{\text {eff }}$ is the effective volume and $V_{0}$ is a normalising factor named reference volume [34].

In order to calculate the effective volumes, the B3B and SPT tests were simulated by finite elements analysis (FEA) models. Then, the tool Abaqus2Matlab [35] was used to obtain the principal stresses and the volume of each element of the specimen at every single frame in the simulation. Knowing the value of these parameters, the total probability of failure, $P_{f_{-} \text {global }}$, can be calculated by $[35,36]$ :

$$
P_{f_{-} \text {global }}=1-\prod\left(1-P_{\text {felement }}\right)
$$

And the effective volume can be expressed as: 


$$
V_{e f f}=\frac{-\ln \left(1-P f_{-} g l o b a l\right) \cdot V_{0}}{\left(\sigma / \sigma_{0}\right)^{m}}
$$

The tool Abaqus2Matlab [35] is most suitable when it is necessary to use finite elements with variable size to model the specimen. This is the case of the considered test configurations in which the biaxial loaded plates are subjected to large stress gradients. In order to accurately simulate the highly stressed central zone, the elements used for its modelling have to be smaller than for the rest of the specimen $[5,18]$.

With Abaqus2Matlab it is possible to extract the specific stress value and the volume of each element and frame from the FEA. The effective volume calculated for large stress gradients strongly depends on the size of these elements. Thus, elements of similar sizes must be used to model the specimens in the different tests in order to make a better comparison between the associated effective volumes.

ABAQUS/Standard 6.12 commercial code was used for the FE analysis. Taking into account the geometrical configurations, a three-dimensional model is necessary for the analysis of stresses in the B3B specimen (Figure 3a) while a simpler two-dimensional axisymmetric model is adequate for the SPT one (Figure 3.b). In order to assure the similarity between the size of the elements in spite of the differences between the models, the same number of elements was used to fill the transversal section of the specimens in both tests (Fig. 3).

The jig elements (punch and matrix for SPT and support and load balls for B3B) were modelled as rigid bodies, while the specimens were modelled as elastic bodies ( $E=100$ $\mathrm{GPa}, v=0.3$ ) using four-node axisymmetric elements with reduced integration (CAX4R) in the case of the SPT and eight-node brick element with reduced integration (C3D8R) elements in the B3B model. A finer mesh was used to model the central zone in both cases in order to have more sensitivity in the results.

Hard contact in the normal direction and frictionless contact in tangential directions between the supporting balls were defined. For both tests, the contact between the specimen and the supporting elements was defined as hard contact in the normal direction and penalty contact, using a friction coefficient of 0.1 [6], in tangential directions. 


\section{Results}

Characteristic load-displacement curves recorded during the tests are plotted in figure 4. In all SPT and B3B curves an initial low stiffness part corresponding to the adjustment of the supports on the specimen was observed for low loads $(<10 \mathrm{~N})$. Then, load increased linearly until the failure load, followed by a sharp load drop in B3B tests (Fig. 4.a). In the SPT curves (Fig. 4.b), the first load drop $\left(P_{1}\right)$ was followed by a load recovery up to a second load drop $\left(P_{2}\right)$ and, in some of the curves, a second recovery up to the failure load, $P_{u}$, occurred. The slope of all the load-displacement curves decreased after $P_{1}$ and $P_{2}$, as corresponds to a decrease of the stiffness of the specimen. In order to identify the processes responsible for the different load drops, additional tests interrupted after $P_{1}$ and $P_{2}$ (4 tests for each load) were done and the tested specimens were analysed. As shown in figure 5, specimens were already divided in two parts after the first load drop, thus, $P_{1}$ was taken as failure load, $P_{1=} P_{C}$.

The fracture surfaces of B3B and SPT specimens had similar features, such as those shown in figure 6. Critical defects were agglomerates (Fig. 6a) and pores associated to differential sintering of agglomerates (Fig. 6b) and fracture was mostly transgranular.

The parameters of the Weibull distributions of the values of $P_{c} / t^{2}$ are shown in table 2. Differences between the characteristic values, $\left(P_{c} / t^{2}\right)_{0}$, are statistically significant while the confidence intervals for the Weibull modulus, $m$, overlap. A value of $m=10$, was used for subsequent calculations.

The values of the characteristic strength, $\sigma_{0}$, calculated from $\left(P_{c} / t^{2}\right)_{0}$ using the different authors proposals (Eqs. 2-5 and 8), together with the limits of the $90 \%$ confidence intervals are summarized in table 3 . It is observed that $\sigma_{0}$ values are independent of the method used to calculate $b$.

For B3B there are no statistically significant differences between the values of $\sigma_{0}$ calculated using the different approaches while SPT results for the equation of Shetty et al. (Eq. 5) are significantly lower than for the other three (Eqs. 2-4), for which the confidence intervals overlap.

Figure 7 plots the Weibull distributions of the strengths computed from the values of $P_{c} / t^{2}$ using the different proposals (Eqs. 2-5 and 8). The distributions calculated using 
$\sigma_{0}$ and $m$ (continuous lines) together with the experimental values and the associated probabilities (Eq. 10, symbols) are shown.

The effective volumes corresponding to the B3B and the SPT tests, calculated using the tool Abaqus2Matlab [35] described above are also included in table 3. Characteristic strength values of 93 and $77 \mathrm{MPa}$ for $\mathrm{B} 3 \mathrm{~B}$ and SPT, respectively, and the same normalising factor, $V_{0}=1 \mathrm{~mm}^{3}$, were used. The B3B effective volume is almost $86 \%$ lower than the SPT one.

\section{Discussion}

Differences between the geometry of the tests are responsible for different stress levels through the specimens for the same applied loads; thus, values of $\left(P_{c} / t^{2}\right)_{0}$ for the two tests (table 2) are different. On the contrary, similar values of the Weibull modulus, $m$, have been found.

The fracture mechanism of the studied material is brittle with strength values determined by microstructural defects, such as those shown in figure 6 , subjected to biaxial stresses in B3B as well as SPT tests. Therefore, even though the actual strength values for each test are different, the critical defects and, consequently, the shapes of the strength distributions are the same, as revealed by the coincidence of $m$ values.

There are no significant differences between the characteristic strength values calculated using the different proposals for B3B (table 3 ) while the value calculated according to Shetty et al. [14] for SPT is lower than the rest. In order to identify the potential origin of such differences, the effect of the radius of the loading ball on the values of the strength calculated through the five analysed equations has been investigated. As shown in figure 8, there is a clear dependence of the calculated strengths with the ball radius. Values of the proposals of Xiong et al., Soltysiak et al. and Timoshenko et al. (Eqs. 2, 3 and 4) coincide through the whole considered interval of ball radius while those of Shetty et al. and Borger et al. (Eqs. 5 and 8) only coincide in the 3 to $5 \mathrm{~mm}$ interval and their values are lower than the others. For ball radii similar to that of the balls used in this work, around $5 \mathrm{~mm}$, all the strength values are coincident. 
The differences between the strength values obtained through the different proposals can be explained based in figure 8. Considering first the SPT results, the small radius of the punch $\left(R_{b}=1.25 \mathrm{~mm}\right)$ leads to the largest differences between the low values for Shetty et al proposal (Eq. 5) and the other ones (Fig. 7 b); differences are enough as to be out of the confidence intervals for the characteristic strength (table 3).

In the case of the B3B results, the use of a larger ball $\left(R_{b}=4.25 \mathrm{~mm}\right)$ leads to values calculated according to Shetty et al and Borger et al values (Eqs. 5 and 8) that are coincident and lower than the other three. In this case, differences are not enough as to give characteristic strength values out of the confidence limits of the different solutions (table 3).

The coincidence of strength values calculated using the equations proposed by Xiong et al, Soltysiak et al and Timoshenko et al (Eqs. 2, 3 and 4) through the wide interval of ball radius considered $(2-7 \mathrm{~mm})$, gives these three proposals a universal character for miniature biaxial strength tests performed with spherical loading elements on small ceramic samples. Furthermore, given that the Timoshenko et al proposal (Eq. 4) does not include the value of radius of the uniform loading central area, $b$, appears that the consideration of this area is not determinant for the calculus, and points out this proposal as the most adequate due to its simplicity.

Taking into account the discussion above, $\sigma_{O_{-} B 3 B}=93$ and $\sigma_{O_{-} S P T}=77 \mathrm{MPa}$ can be identified as characteristic strength values for B3B and SPT, respectively. The large difference between these values $\left(\sigma_{O B 3 B} / \sigma_{O S P T} \approx 1.2\right)$ is coincident with what is predicted by the well-known Weibull relationship given by Eq. 14 and [34] for the effective volumes associated to the test configurations (table 3 ) and the Weibull modulus of the material, $m$.

$$
\frac{\sigma_{0 \_} S P T}{\sigma_{0 \_B 3 B}}=\left(\frac{V_{e f f \_B 3 B}}{V_{e f f \_S P T}}\right)^{1 / m}
$$




\section{Conclusions}

In this work the fracture behaviour of commercial discs of soft PZTs tested using two different biaxial miniature test configurations (B3B and SPT) has been analysed and the characteristic strength values of both tests have been calculated using different approaches.

The validity of SPT for characterizing strength of PZT has been proved. Results are comparable to those obtained by $\mathrm{B} 3 \mathrm{~B}$ using the Weibull relationship for effective volumes.

Timoshenko's proposal (Eq. 4) has been identified as the most adequate for calculation the strength values in the case of miniature biaxial strength tests done with spherical loading elements of radius between 2 and $7 \mathrm{~mm}$.

\section{Acknowledgements}

Projects MAT2014-58738-C3 and CSIC PIE 201660E097C. The authors would like to thank to the Principado de Asturias government for the financing support given to the IDI/2018/000134 project. C. Quintana also thanks the financial support from the Principado de Asturias Regional Government through the Severo Ochoa Scholarship Programme (contract BP17-27 QBC).

\section{REFERENCES}

[1] D.C. Lupascu, J. Rödel, Fatigue in bulk lead zirconate titanate actuator materials, Adv. Eng. Mater. 7. (2005) 882-898.

[2] S. Saadon, O. Sidek, A review of vibration-based MEMS piezoelectric energy harvesters, Energy Convers. Manag. 52 (2011) 500-504.

[3] T.Y. Zhang, C.F. Gao, Fracture behaviors of piezoelectric materials, Theor. Appl. Fract. Mech. 41 (2004) 339-379.

[4] R. Fu, C.F. Qian, T.Y. Zhang, Electrical fracture toughness for conductive cracks driven by electric fields in piezoelectric materials, Appl. Phys. Lett. 76:1 (2000) 126-128.

[5] A. Börger, P. Supancic, R. Danzer, The ball on three balls test for strength testing 
of brittle discs: Stress distribution in the disc, J. Eur. Ceram. Soc. 22 (2002) 14251436.

[6] A. Börger, P. Supancic, R. Danzer, The ball on three balls test for strength testing of brittle discs: Part II: Analysis of possible errors in the strength determination, J. Eur. Ceram. Soc. 24 (2004) 2917-2928.

[7] R. Danzer, W. Harrer, P. Supancic, T. Lube, Z. Wang, A. Börger, The ball on three balls test-Strength and failure analysis of different materials, J. Eur. Ceram. Soc. 27 (2007) 1481-1485.

[8] S. Rasche, S. Strobl, M. Kuna, R. Bermejo, T. Lube, Determination of Strength and Fracture Toughness of Small Ceramic Discs Using the Small Punch Test and the Ball-on-three-balls Test, Procedia Mater. Sci. 3 (2014) 961-966.

[9] H. Zielke, M. Abendroth, M. Kuna, Determining fracture mechanical properties for brittle materials using the ball on three balls test combined with numerical simulations, Theor. Appl. Fract. Mech. $86 \quad$ (2016) 19-24. doi:10.1016/j.tafmec.2016.09.001.

[10] A.F. Kirstein, R.M. Woolley, Symmetrical Bending of Thin Circular Elastic Plates on Equally Spaced Point Supports, J. Res. Natl. Bur. Stand. C. Eng. Instrum. 71C:1 (1967) 1-10.

[11] Z. Xiong, W. Jiang, Y. Shi, A. Kawasaki, R. Watanabe, Evaluation of hightemperature strength of Mo/PSZ composites by modified small punch tests, Mater. Trans. 46:3 (2005) 631-636.

[12] S. Soltysiak, M. Abendroth, M. Kuna, Y. Klemm, H. Biermann, Strength of fine grained carbon-bonded alumina (Al2O3-C) materials obtained by means of the small punch test, Ceram. Int. 40 (2014) 9555-9561.

[13] S.P. Timoshenko, S. Woinowsky-Krieger, Theory of plates and shells, 2nd Edit, McGraw- Hill International Editions, New York, 1959.

[14] D.K. Shetty, A.R. Rosenfield, W.H. Duckworth, P.R. Held, A Biaxial-Flexure Test for Evaluating Ceramic Strengths, J. Am. Ceram. Soc. 66 (1983) 36-42.

[15] H.M. Westergaard, Stresses in concrete pavements computed by theoretical analysis, Public Roads. 7 (1926) 25-35. 
[16] D.J. Godfrey, Fabrication, formulation, mechanical properties, and oxidation of sintered Si 3 N 4 ceramics using disc specimens, Mater. Sci. Technol. 1:7 (1985) $510-515$.

[17] M.P. Manahan, A.S. Argon, O.K. Harling, The development of a miniaturized disk bend test for the determination of postirradiation mechanical properties, J. Nucl. Mater. 104 (1981) 1545-1550.

[18] T.E. García, C. Rodríguez, F.J. Belzunce, C. Suárez, Estimation of the mechanical properties of metallic materials by means of the small punch test, J. Alloys Compd. 582 (2014) 708-717.

[19] M. Fernández, C. Rodríguez, F.J. Belzunce, T.E. García, Use of small punch test to estimate the mechanical properties of powder metallurgy products employed in the automotive industry, Powder Metall. 58:3 (2015) 171-177.

[20] C. Rodríguez, J. Cabezas, E. Cardenas, F.J. Belzunce, C. Betegon, Mechanical Properties Characterization of Heat-Affected Zones Using the Small Punch Test, Weld. J. 88 (2009) 188-192.

[21] A. Janča, J. Siegl, P. Haušild, Small punch test evaluation methods for material characterisation, J. Nucl. Mater. 481 (2016) 201-213.

[22] C. Rodríguez, D. Arencón, J. Belzunce, M.L. Maspoch, Small punch test on the analysis of fracture behaviour of PLA-nanocomposite films, Polym. Test. 33 (2014) 21-29.

[23] C. Rodríguez, I.I. Cuesta, M.L.L. Maspoch, F.J. Belzunce, Application of the miniature small punch test for the mechanical characterization of polymer materials, Theor. Appl. Fract. Mech. 86 (2016) 78-83.

[24] C. Rodríguez, M. Fernández, J.G. Cabezas, T.E. García, F.J. Belzunce, The use of the small punch test to solve practical engineering problems, Theor. Appl. Fract. Mech. 86 (2016) 109-116.

[25] S. Hackemann, W. Pfeiffer, Domain switching in process zones of PZT: characterization by microdiffraction and fracture mechanical methods, J. Eur. Ceram. Soc. 23:1 (2003) 141-151.

[26] M. Selten, G.A. Schneider, V. Knoblauch, R.M. McMeeking, On the evolution of 
the linear material properties of PZT during loading history-an experimental study, Int. J. Solids Struct. 42:13 (2005) 3953-3966.

[27] T. Tanimoto, K. Okazaki, K. Yamamoto, Tensile stress-strain behavior of piezoelectric ceramics, Jpn. J. Appl. Phys. 32:9 (1993) 4233-4236.

[28] W. Hooker, Properties Ceramics of PZT-Based Piezoelectric ceramics between 150 and $250^{\circ} \mathrm{C}, \mathrm{NASA} / \mathrm{CR}-208708$. (1998).

[29] C.S. Watson, Strength and Reliability of Lead Zirconate Titanate Ceramics, Fract. Mech. Ceram. 14 (2005) 485-498.

[30] P. Supancic, Z. Wang, W. Harrer, K. Reichmann, R. Danzer, Strength and Fractography of Piezoceramic Multilayer Stacks, Key Eng. Mater. 290 (2005) 4653.

[31] UNE-EN 843-5:2006. Advanced technical ceramics, (2006).

[32] W. Weibull, A Statistical Distribution Function of Wide Applicability, J. Appl. Mech. 18 (1951) 293-297.

[33] W. Harrer, R. Danzer, P. Supancic, T. Lube, Influence of the Sample Size on the Results of B3B-Tests, Key Eng. Mater. 409 (2009) 176-184.

[34] K. Trustrum, A. Jayatilaka., On estimating the Weibull modulus for a brittle material, J. Mater. Sci. 14 (1979) 1080-1084.

[35] G. Papazafeiropoulos, M. Muñiz-Calvente, E. Martínez-Pañeda, Abaqus2Matlab: A suitable tool for finite element post-processing, Adv. Eng. Softw. 105 (2017) 916.

[36] S.L. Fok, B.C. Mitchell, J. Smart, B.J. Marsden, A numerical study on the application of the Weibull theory to brittle materials, Eng. Fract. Mech. 68:10 (2001) 11711179. 
FIGURE CAPTIONS

a)
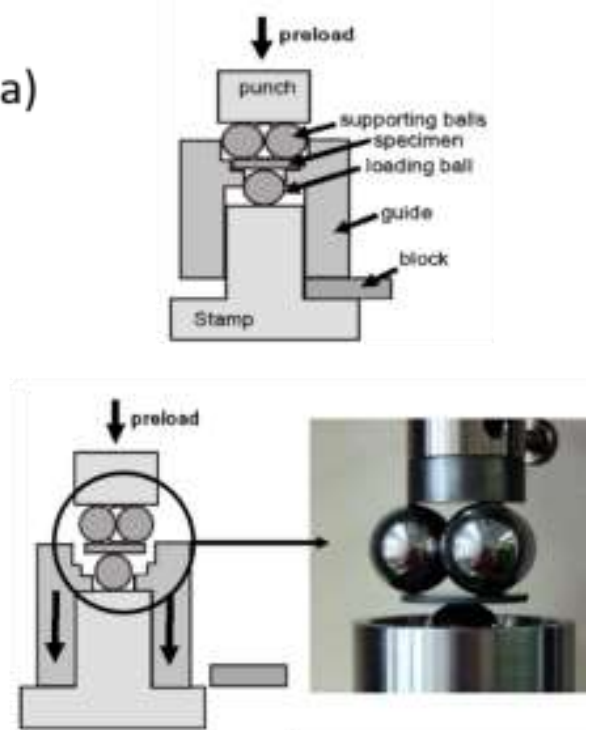

b)

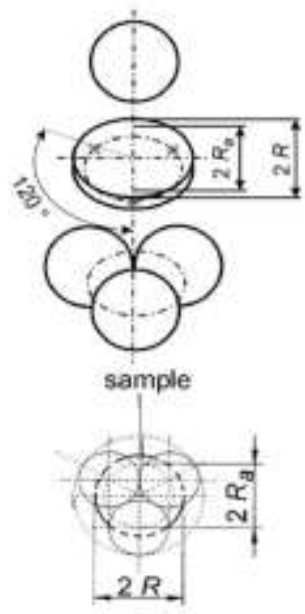

Figure 1: Scheme of the experimental set-up of the B3B test [6]
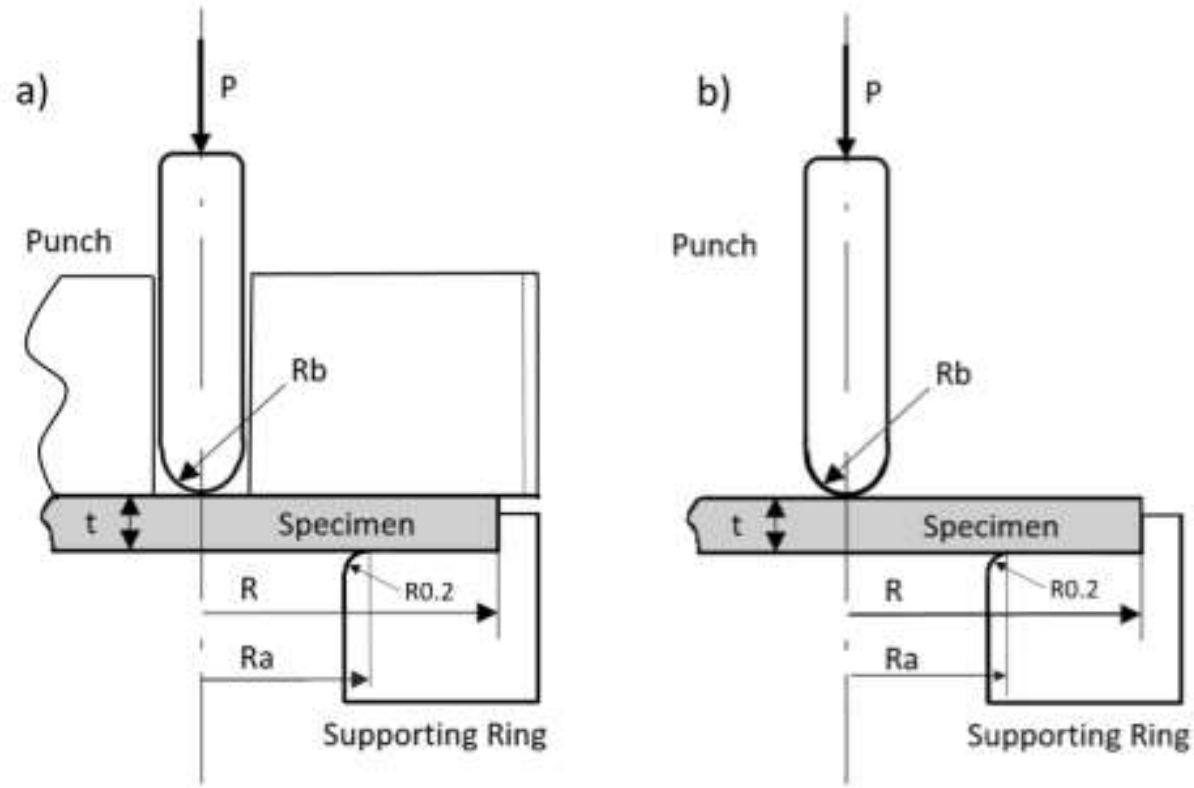

Figure 2: Scheme of the SPT configuration: a) for metallic materials; b) for ceramic materials 

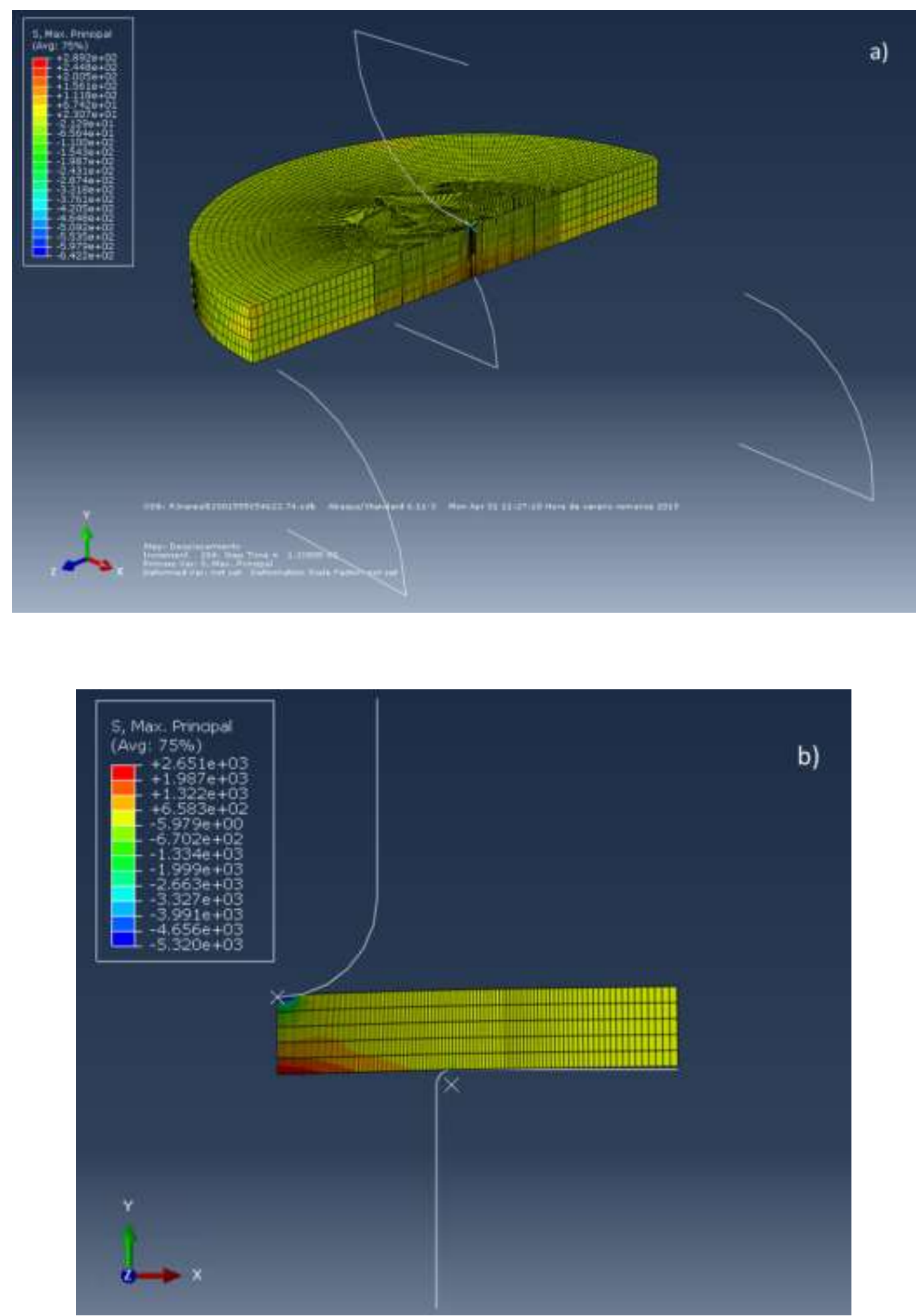

Figure 3: FEA models and maximum principal stress on the tensile side of the discs

$$
\text { ( } F=100 N) \text { of: a) B3B test and b) SPT }
$$



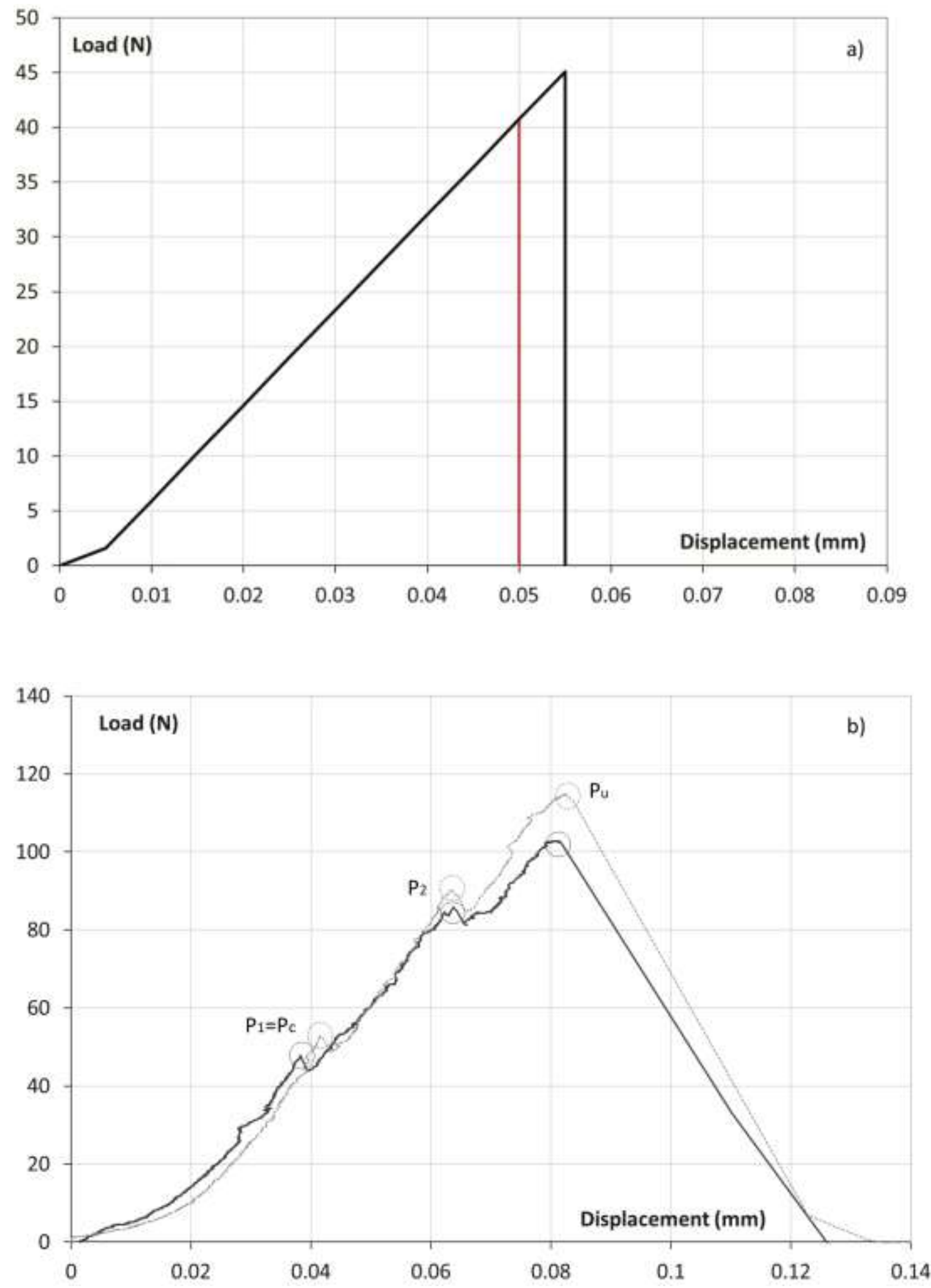

Figure 4: Typical experimental load-LPD curves: a) B3B test; b) SPT. The different load drops are indicated. 


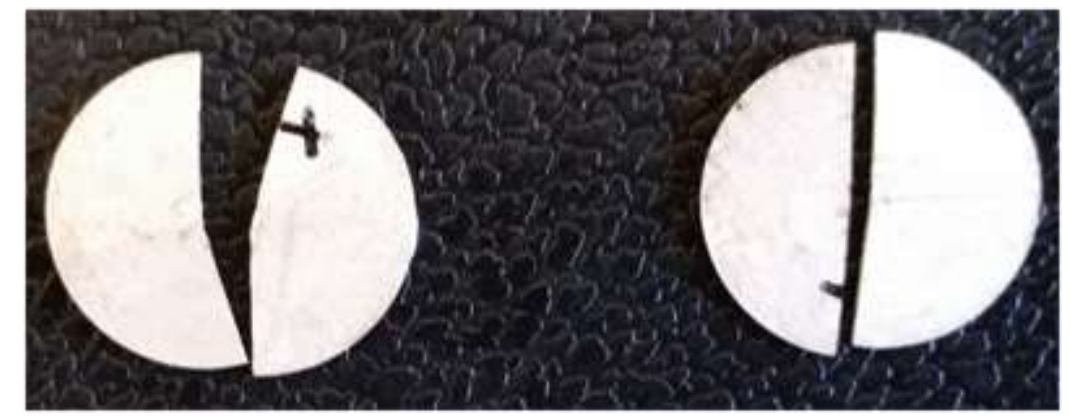

Figure 5: Appearance of two samples which tests were stopped just after the first load drop at $P_{1}$. 
a)

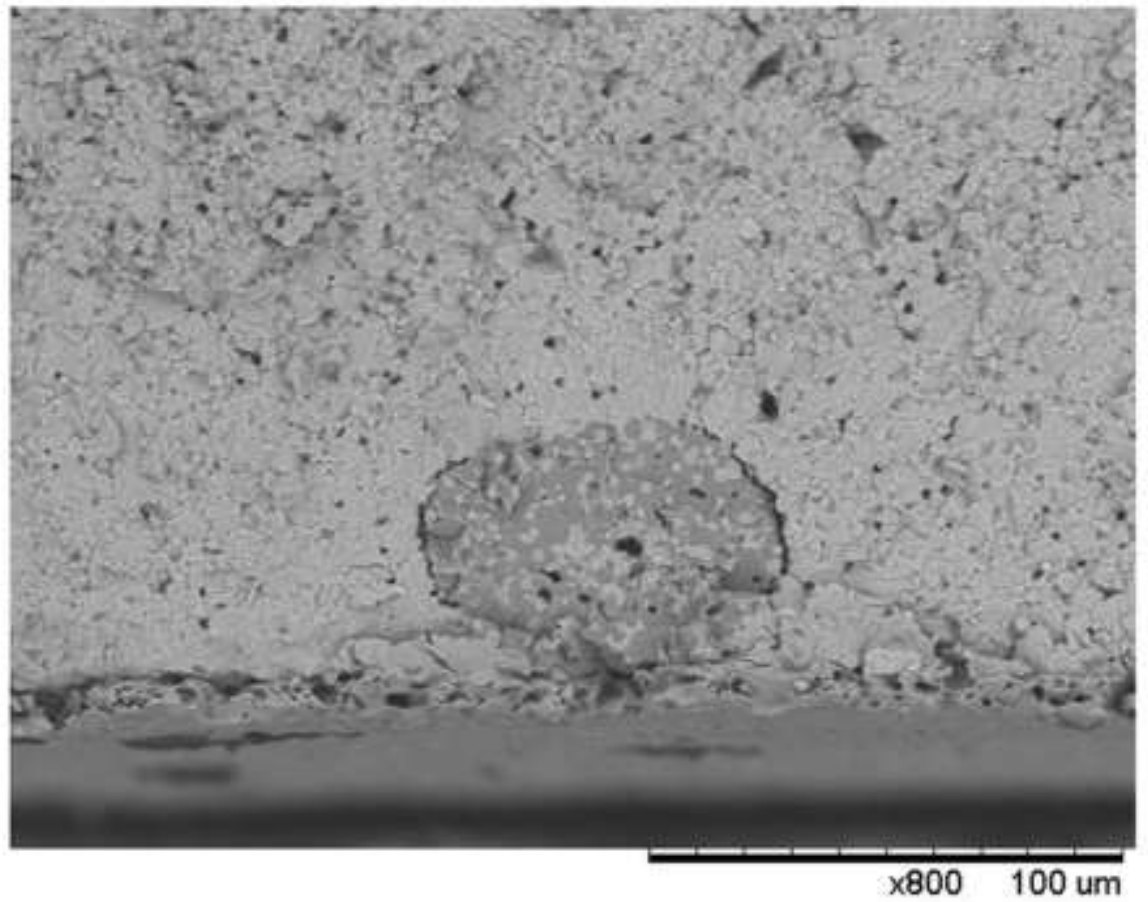

b)

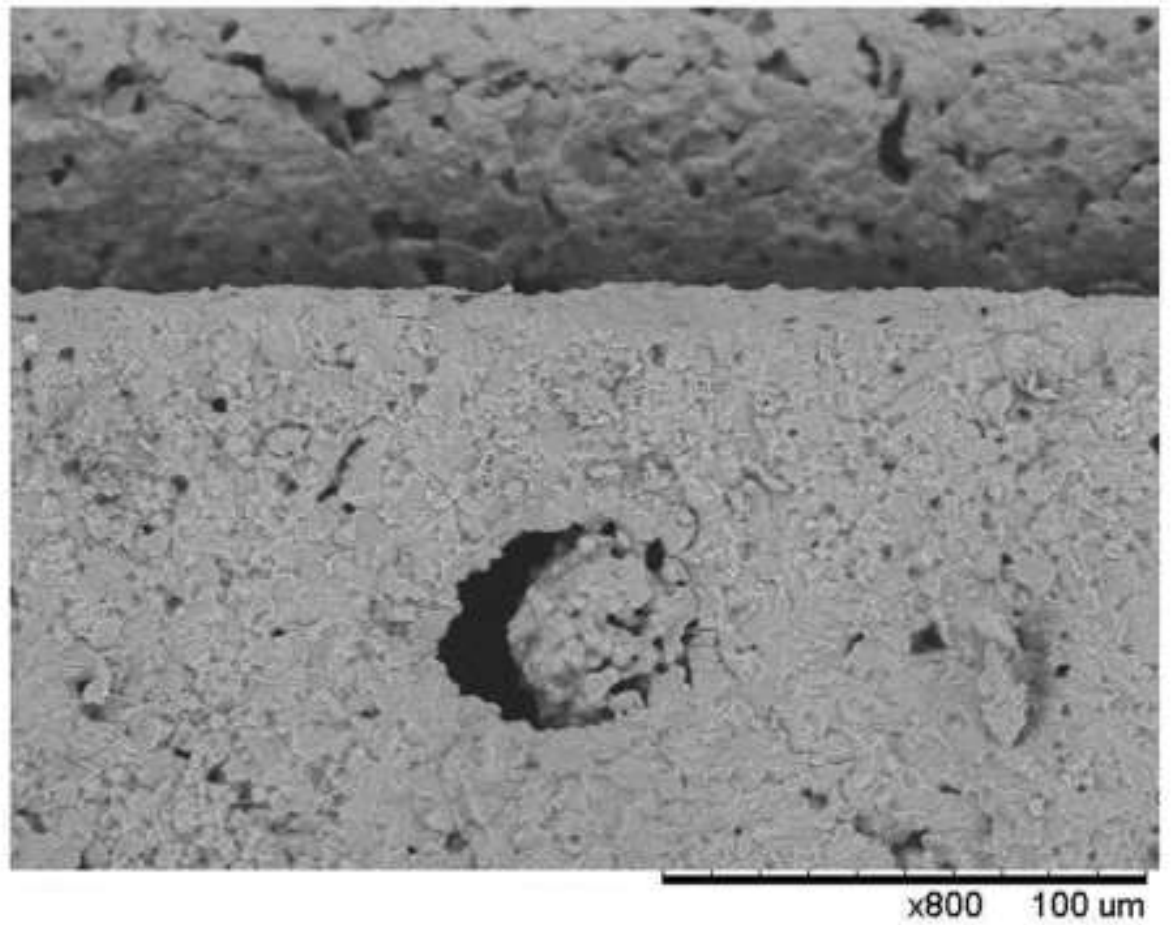

Figure 6: Characteristic critical defects found in the fracture surfaces: a)

Agglomerate; b) Pore associated to differential sintering of an agglomerate. 

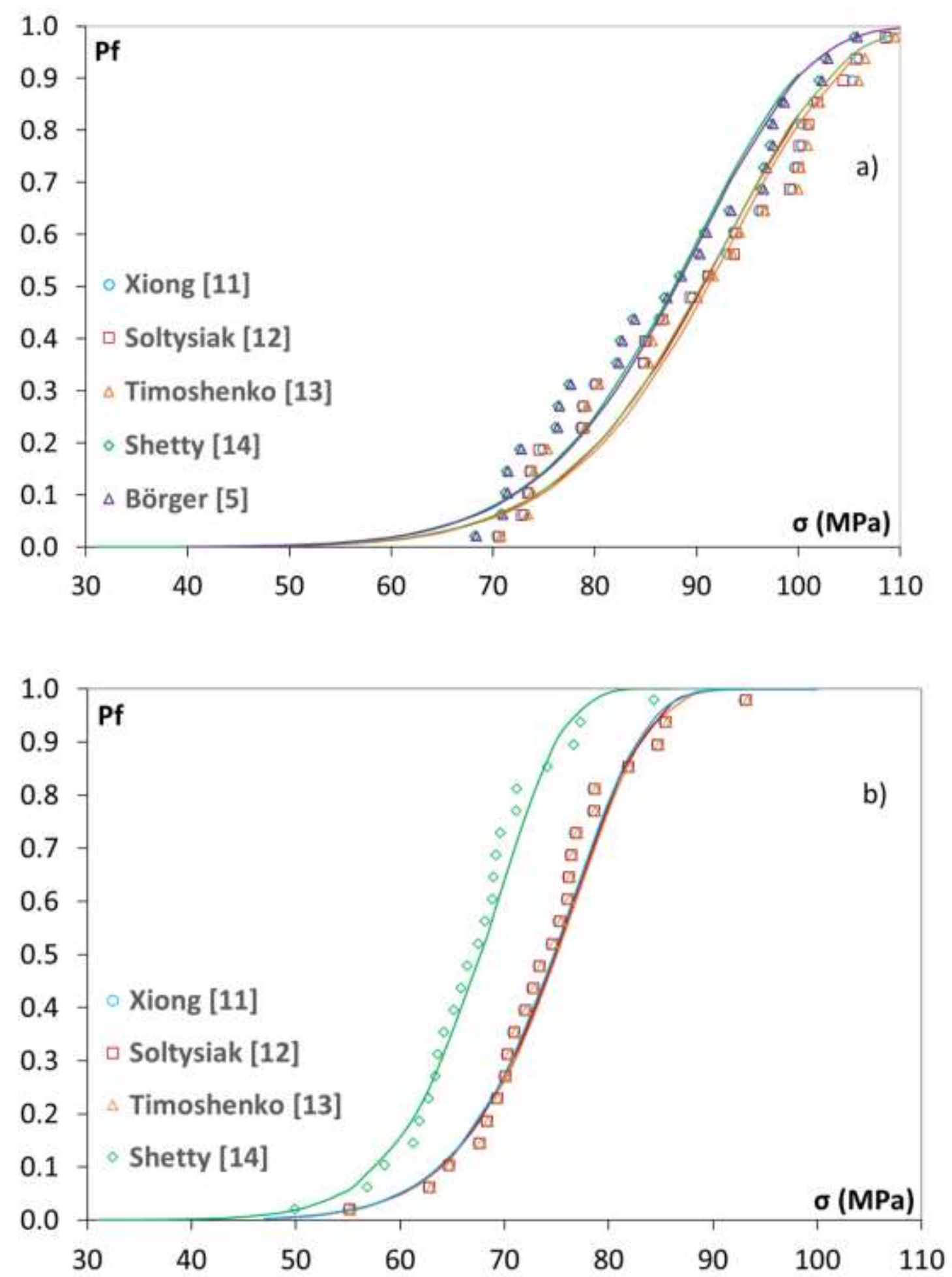

Figure 7: Weibull statistical distribution of strengths obtained using the indicated equations. Value of the b-parameter is calculated according to Shetty et al. [14]: a) B3B test; b) SPT. 


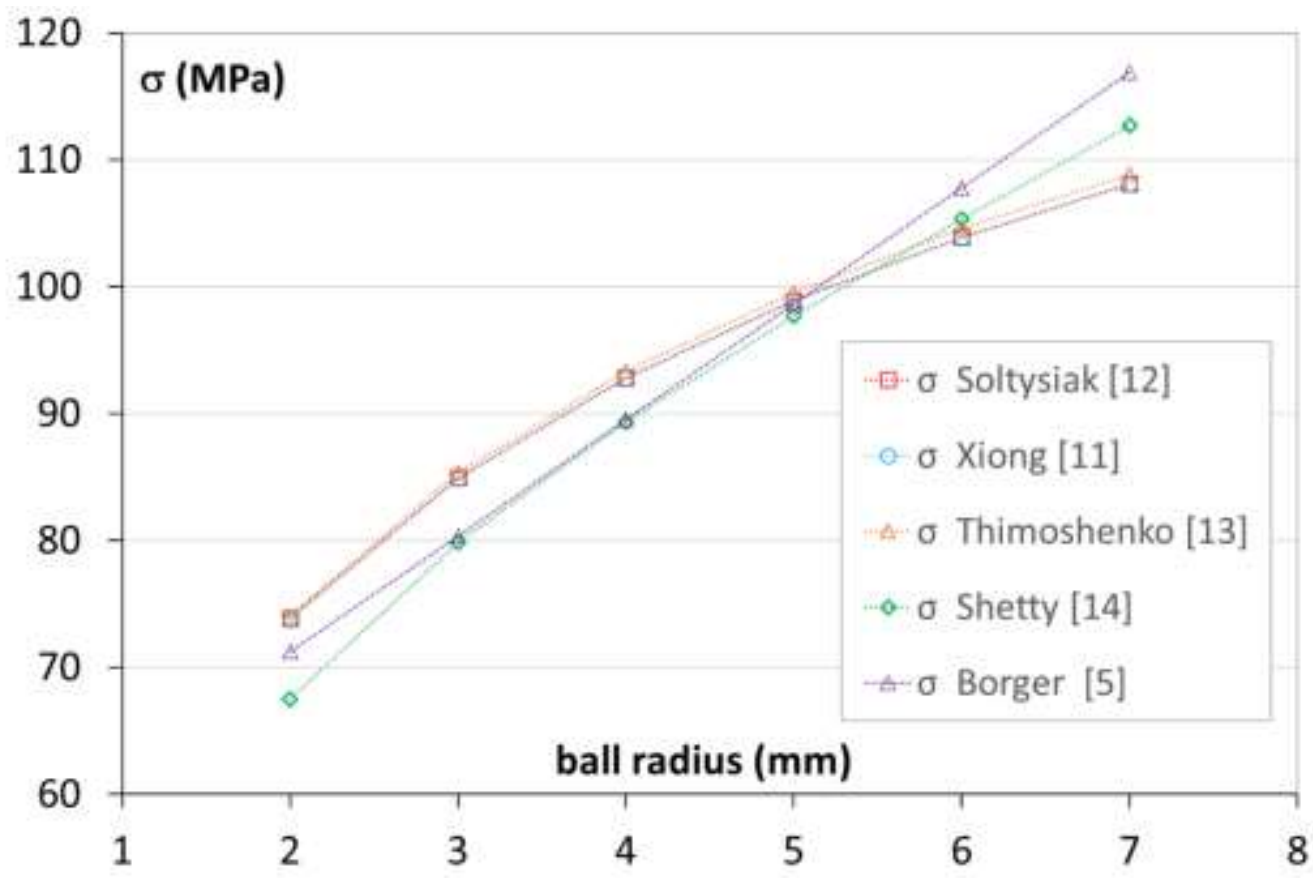

Figure 8: Influence of the loading ball radius of the B3B test on the calculated tensile stress for different approximations. 
TABLES

Table 1. Proposals to calculate the radius of the central loaded area assumed subjected to a constant stress value, $b$.

\begin{tabular}{c|ll} 
& \multicolumn{2}{|c}{$b$} \\
\hline Shetty et al. [14] & $t / 3$ & For $\zeta \geq 0$ \\
\hline Westergaard [15] & $t$ & For $\zeta>1.724 t$ \\
\cline { 2 - 3 } & $\left(1.6 \zeta^{2}+t^{2}\right)^{1 / 2}-0.675 t$ & For $0 \leq \zeta<1.724 t$ \\
\hline
\end{tabular}

Table 2. Weibull modulus, $m$, and characteristic value, $\left(\boldsymbol{P}_{c} / t^{2}\right)_{0}$, of the distributions of $\boldsymbol{P}_{c} / \boldsymbol{t}^{2}$ values. The values between brackets are the lower and upper limits for $90 \%$ confidence limits.

\begin{tabular}{lccc}
\hline Type of test & $\begin{array}{c}\text { Number of tested } \\
\text { specimens }(\boldsymbol{n})\end{array}$ & $\begin{array}{c}\left(\boldsymbol{P}_{\boldsymbol{c}} / \mathbf{t}^{\mathbf{2}}\right)_{0} \\
(\mathrm{MPa})\end{array}$ & $\boldsymbol{m}$ \\
\hline B3B & 24 & $\begin{array}{c}43.9 \\
{[42-45.4]}\end{array}$ & $\begin{array}{c}9.4 \\
{[7-12]}\end{array}$ \\
\hline \multirow{2}{*}{ SPT } & 24 & 48.6 & 11.8 \\
& & {$[47-50.1]$} & {$[9-15]$} \\
\hline
\end{tabular}

